Gillian T. Barzaga, MD

Department of Otorhinolaryngology Head and Neck Surgery

De La Salle University Medical Center

Correspondence: Dr. Gillian T. Barzaga Department of Otorhinolaryngology Head and Neck Surgery

De La Salle University Medical Center De La Salle Health Sciences Institute

Gov. D. Mangubat Avenue, Dasmariñas Cavite 4114

Philippines

Phone: (632) 9162076401

E-mail address: gtbarzaga@dlshsi.edu.ph

The author declared that this represents original material that is not being considered for publication or has not been published or accepted for publication elsewhere or in part, in print or electronic media; that the requirements for authorship have been met by the author, and the author believes that the manuscript represents honest work.

Disclosures: The author signed a disclosure that there are no financial or other (including personal) relationship, intellectual passion, political or religious beliefs, and institutional affiliations that might led to conflict of interest.

\section{The Relationship of Surgeon Handedness and Experience on Operative Duration and Hearing Improvement in Ipsilateral and Contralateral Otologic Surgeries}

\begin{abstract}
Objective: To determine the relationship of the surgeon handedness and operative site laterality on operative duration and hearing improvement in otologic surgery, and to further explore whether this relationship may be related to surgeon experience.
\end{abstract}

\section{Methods:}

\section{Design: Retrospective Cohort \\ Setting: $\quad$ Tertiary Private Teaching Hospital}

Participants: Seventy-three (73) patients aged 18 to 65 years old who underwent primary ear surgery under general anesthesia between January 2016 and December 2019 were retrospectively divided into two groups: 39 contralateral and 34 ipsilateral. The operative durations and hearing improvements were compared using independent t-tests, with consideration of surgeon experience in years further stratifying patients.

Results: There was no significant difference in operative duration, $t(71)=1.14, p=.26$, between the contralateral $(M=281.95$ minutes, $S D=71.82)$ and ipsilateral $(M=261.15, S D=79.26)$ groups. This same pattern was more pronounced among surgeons with $10+$ years of experience although there was also no significant difference in operative time, $\mathrm{t}(33)=1.31, \mathrm{p}=.19$ for both ipsilateral and contralateral surgeries There was no statistically significant difference, $t(36)=-0.72, p=.47$, in overall mean hearing gain among patients in the contralateral $(M=+2.22 \mathrm{~dB}, \mathrm{SD}=10.54)$ and ipsilateral $(M=+5.12 \mathrm{~dB}, \mathrm{SD}=14.26)$ groups. Although the difference was also not statistically significant, $\mathrm{t}(16)=-1.94, \mathrm{p}=.07$ for contralateral $(\mathrm{M}=0.00, \mathrm{SD}=5.43)$ and ipsilateral $(\mathrm{M}=+7.95$ $\mathrm{dB}, \mathrm{SD}=11.52$ ) procedures performed by surgeons with experience of 10 years or more, a mean hearing gain of $+7 \mathrm{~dB}$ in the ipsilateral group compared to $0 \mathrm{~dB}$ in the contralateral group was notable.

Conclusion: This study did not prove that regardless of surgeon experience, right-handed surgeons operating on the right ear and left-handed surgeons operating on the left ear have better ear surgery outcomes of operative duration and hearing improvement compared to righthanded surgeons operating on the left ear and left-handed surgeons operating on the right ear. Future studies on larger samples with more complete data may yet demonstrate this effect.

Keywords: handedness; laterality; otologic surgical procedure; hearing; operative times. 
Most people all over the world are right-handed $(90 \%) ;{ }^{1}$ and this is reflected among surgeons, where left-handed individuals represent only $9-10 \%{ }^{2}$ Surgeon handedness may be related to optimal surgical performance, and their inherent dominant hand may contribute to this performance depending on the laterality of the surgical field. Very few studies on orthopedics, ${ }^{3,4}$ breast surgery, ${ }^{5}$ and microlaryngeal surgery ${ }^{6}$ document the relationship of handedness and laterality of operative site with positive outcomes. A study of a right-handed orthopedic surgeon operating on knees of different literalities found that the odds of having a poor outcome were higher when operating on the contralateral side. ${ }^{3}$ Another study found that surgeons performing total hip replacements were able to increase the angulation of the joint socket more easily when operating ipsilateral to their dominant hand, restoring the hip nearer to its normal anatomic position. ${ }^{4}$ Breast surgeons have no significant differences in terms of complication when operating on either breast, but it was found that less experienced surgeons had significantly more complications than experienced ones. ${ }^{5}$ To the best of my knowledge, there are no published reports so far on the effect of surgeon handedness on otologic surgery.

Do right-handed surgeons operating on the right ear and lefthanded surgeons operating on the left ear have an advantage in ear surgery outcomes such as operative duration and hearing improvement compared to right-handed surgeons operating on the left ear and lefthanded surgeons operating on the right ear of the patient? Would surgeon experience contribute to the effect of such surgeon-patient positioning?

Hypothesizing that operative duration should be shorter and hearing gain will be better when operative ears are ipsilateral to the surgeon's dominant hand, and that these outcomes may be further improved with surgeon experience greater than ten years, this research aims to determine the difference in the operative duration and hearing improvement between patients whose surgical site was ipsilateral the surgeon's dominant hand compared to those whose surgical site was contralateral the surgeon's dominant hand, and to further explore whether this relationship may be related to surgeon experience.

\section{METHODS}

This retrospective cohort study reviewed records of patients aged 18-65 years who underwent primary ear surgery under general anesthesia in a tertiary private teaching hospital between January 1 , 2016 and December 31, 2019. The study was approved by the De La Salle Health Sciences Institute Independent Ethics Committee [DLSHSIIEC(2019)-52-02-A]. Informed consent was waived by the board.
The list of otologic surgeries performed on adult patients was generated from the departmental census, and records of operations were retrieved from the hospital medical records section. The following variables were extracted: age, sex, otologic procedure performed and post-operative diagnosis, laterality, start and end times of operation, and intraoperative ossicular findings. Audiograms were retrieved from the institutional hearing center when they were not found in individual clinical records. The name of the surgeon was listed, and their dominant hand and experience in years ( $<10$ or 10 or more) were confirmed and tabulated by the author. Records of patients with incomplete demographic and operative data were excluded. Records with incomplete audiograms were only excluded from hearing gain outcome computations.

The primary outcome was operative duration measured in minutes, while the secondary outcome was hearing gain, measured by the preoperative and post-operative air-bone gap difference. The two groups were additionally compared with regard to surgeon experience.

The patients were classified into two groups: contralateral, C (righthanded surgeons operating on the left ear; left-handed surgeons operating on the right ear) and ipsilateral, I (right-handed surgeons operating on the right ear; left-handed surgeons operating on the left ear). Operative duration was measured in minutes. Hearing gain was computed from the difference between pre- and post-operative airbone gaps in pure-tone audiograms. The groups were further stratified based on surgeon experience in years ( $<10$ vs. 10 or more).

The mean time duration of ear surgeries (regardless of handedness and laterality) in the institution for the year 2018 was 282 minutes, with procedures operated ipsilateral to the dominant hand of the surgeon lasting 230 minutes while those on the contralateral side lasting 290 minutes. Assuming these means with a standard deviation of 89 minutes, a power of $80 \%$ and a $5 \%$ level of significance, the sample size calculated was 35 per group, or a total of 70 subjects using the formula:

$$
n_{A}=\kappa n_{B} \text { and } n_{B}=\left(1+\frac{1}{\kappa}\right)\left(\sigma \frac{z_{1-\alpha / 2}+z_{1-\beta}}{\mu_{A}-\mu_{B}}\right)^{2}
$$

The data was collated and analyzed by the investigator in MS Excel for Mac v.16.33 (2019, Microsoft Corp., Redmond, WA). Descriptive measures (mean, standard deviation) were used and inferential statistics included a two-sample t-test assuming equal variances with a $95 \%$ confidence level and a level of significance of 0.05 to compare the mean difference of patients in the two groups that were considered as independent samples. 


\section{RESULTS}

A total of 105 records of patients who underwent ear surgery in our institution between January 1, 2016 and December 31, 2019 were examined for eligibility. After applying inclusion and exclusion criteria, records of 73 patients were included in the study for operative time outcome. Mean age was 38 years ( 18 to 65 years old). There were 39 patients in the contralateral (C) group and 34 in the ipsilateral (I) group with a male-to-female ratio of 15:24 and 14:20, respectively. Table 1 shows the diagnoses of the patients sorted by group.

Out of the 73 patients included in the study, only 38 had complete pre-operative and post-operative audiograms: 22 in the contralateral (C) group, 16 in the ipsilateral (I) group. Distribution according to hearing level is shown in Figure 1 for the $\mathrm{C}$ group, and Figure 2 for the I group.

Table 1. Demographics of disease and complications

\begin{tabular}{|c|c|c|}
\hline \multicolumn{1}{|c|}{ Diagnoses } & Contralateral & Ipsilateral \\
\hline Chronic otitis media & $\mathbf{3 7}$ & $\mathbf{3 4}$ \\
\hline Cholesteatoma & 14 & 17 \\
\hline Facial nerve dehiscence & 1 & 0 \\
\hline Facial paralysis & 2 & 2 \\
\hline Labyrinthine fistula & 1 & 1 \\
\hline Mastoiditis & 0 & 1 \\
\hline Intracranial extension & 0 & 1 \\
\hline No complications & 19 & 12 \\
\hline Congenital cholesteatoma & $\mathbf{1}$ & $\mathbf{0}$ \\
\hline $\begin{array}{c}\text { Tympanic Membrane perforation } \\
\text { secondary to chemical burn }\end{array}$ & $\mathbf{1}$ & $\mathbf{0}$ \\
\hline Grand Total & $\mathbf{3 9}$ & $\mathbf{3 4}$ \\
\hline
\end{tabular}

Figure 1 shows the pre- and post-operative hearing level classifications of the patients in the contralateral (C) group. Among the 22 patients, the majority had pre-operative mild (8) hearing loss, followed by profound (5), moderate (4) and severe (1) hearing loss, and normal hearing levels, (4). Post-operatively, most patients had moderate hearing loss (8), followed by profound (5), mild (4), severe (3), and normal (2) hearing levels.

Figure 2 shows the pre- and post-operative hearing level classifications of the patients in the ipsilateral (I) group. Among the 16 patients, the majority pre-operatively had moderate hearing loss (6), followed by severe (5), profound (3), mild (1) hearing loss, and normal hearing (1). Post-operatively, most patients had profound (4), severe (4), and mild (4), followed by moderate (2) hearing loss, and normal hearing (2).

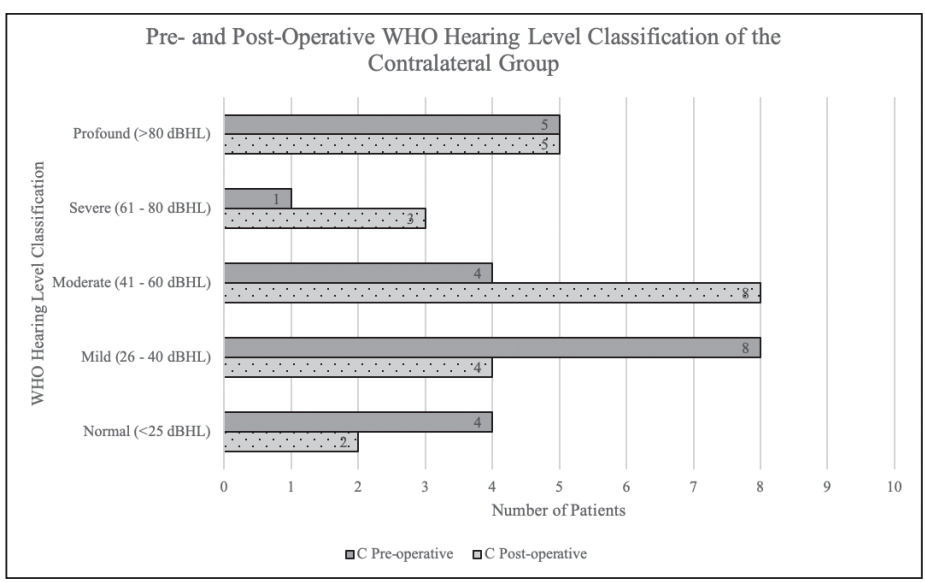

Figure 1. Pre- and post-operative World Health Organization (WHO) hearing level classifications of patients in the contralateral group: superior solid bars, pre-operative; inferior stippled bars, postoperative.

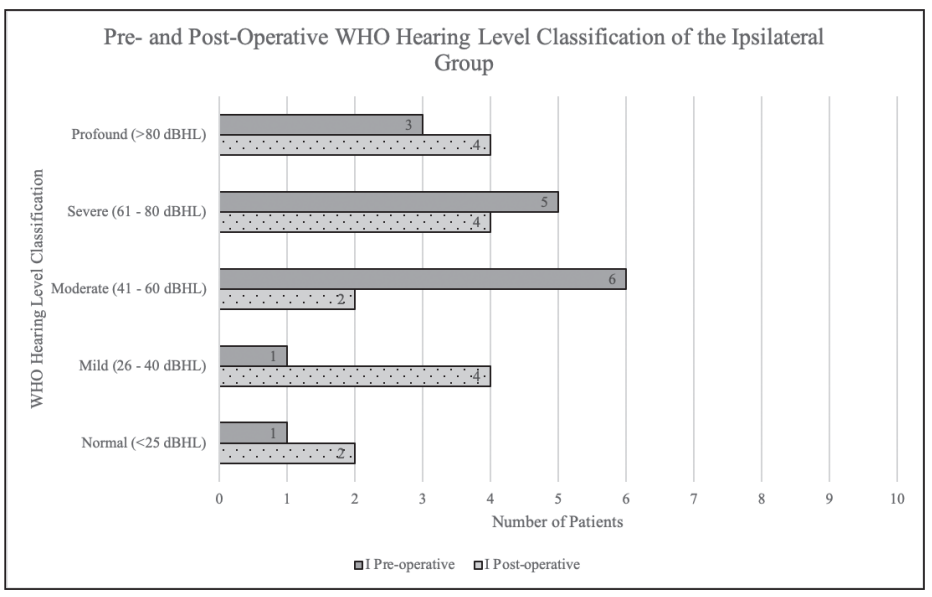

Figure 2. Pre- and post-operative World Health Organization (WHO) hearing level classifications of patients in the ipsilateral group: superior solid bars, pre-operative; inferior stippled bars, postoperative.

Figure 3 shows the otologic procedures performed. For both contralateral (C) and ipsilateral (I) surgeries respectively, the majority $(C=17 ; \quad I=14)$ were canal wall down mastoidectomies with tympanoplasties $(C W D+T)$, followed by intact canal wall mastoidectomies with tympanoplasties $(I C W+T, C=11 ; I=11)$, radical mastoidectomies (RM, $C=5 ; \mathrm{l}=5$ ) and tympanoplasties ( $\mathrm{T}, \mathrm{C}=6 ; \mathrm{I}=5)$.

Table 2 lists the intraoperative ossicular status of the patients. Out of 73 patient records, 63 reported the status of the ossicles: 35 from the contralateral group, 28 from the ipsilateral group. Most patients had intact ossicular chains $(C=15 ; \mid=10)$ followed by those with partially eroded malleus and incus but intact stapes $(C=5 ; l=5)$, those with absent ossicles $(C=4 ; I=2)$, those with partially eroded malleus but intact incus and stapes $(C=3 ; l=3)$ and solely absent incus $(C=3 ; l=3)$, those with solely present stapes $(C=2 ; I=2)$, then those with absent 


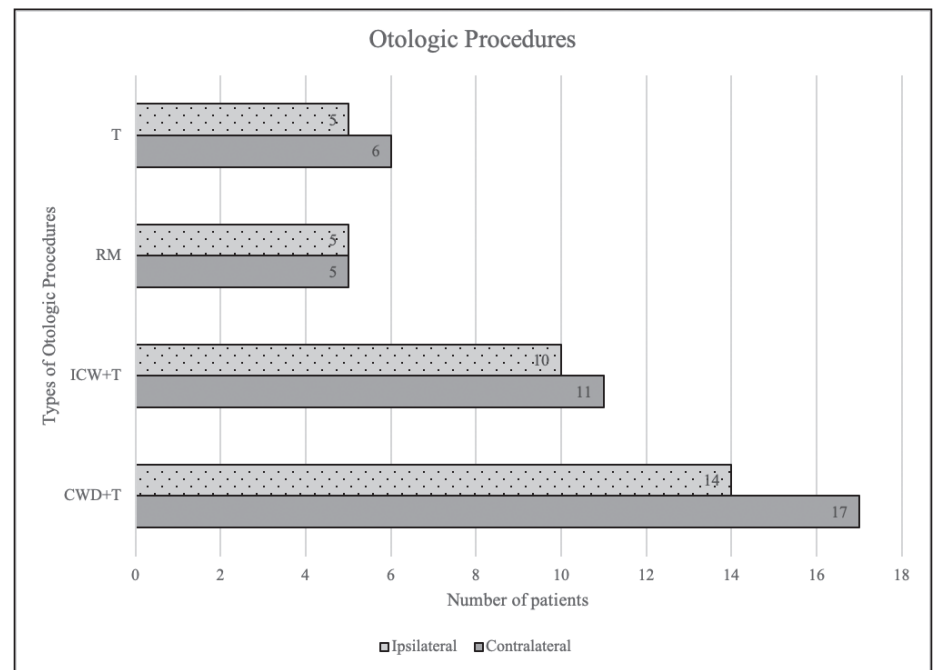

Figure 3. Otologic procedures performed: $T$, tympanoplasty; RM, radical mastoidectomy; ICW+T, intact canal wall mastoidectomy with tympanoplasty; CWD+T, canal wall down mastoidectomy with tympanoplasty: superior stippled bars, ipsilateral; inferior solid bars, contralateral.

Table 2. Ossicular chain status of the patients

\begin{tabular}{|c|c|c|}
\hline Ossicular Status + & Contralateral & Ipsilateral \\
\hline$M+I+S+$ & 15 & 10 \\
\hline$M p l+S+$ & 3 & 3 \\
\hline$M+l-S+$ & 3 & 3 \\
\hline$M p l p S+$ & 5 & 5 \\
\hline$M-I p S+$ & 1 & 1 \\
\hline$M-I-S+$ & 2 & 2 \\
\hline$M p l-S p$ & 1 & 1 \\
\hline MplpS- & 1 & 1 \\
\hline M-I-S- & 4 & 2 \\
\hline Grand Total & $\mathbf{3 5}$ & $\mathbf{2 8}$ \\
\hline
\end{tabular}

†M, malleus; I, incus; S, stapes; +, present; - , absent; $p$, eroded

malleus, partially eroded incus but intact stapes $(C=1 ; I=1)$, absent incus but partially eroded malleus and stapes $(C=1 ; I=1)$, and partially eroded malleus and incus but intact stapes $(C=1 ; l=1)$.

There was no significant time difference in operative duration, $t(71)=1.14, p=.26$ between the 39 patients in the contralateral group $(\mathrm{M}=281.95$ minutes, $\mathrm{SD}=71.82)$ compared to the 34 patients in the ipsilateral group $(M=261.15, S D=79.26)$, even if the ipsilateral group had a shorter operative duration by 20 minutes. There was also no significant difference in operative time, $t(36)=0.09, p=.93$ for surgeon experience of less than 10 years for both the contralateral $(M=265.59$ minutes, $S D=87.63)$ and ipsilateral $(M=263.24$ minutes, $S D=65.28)$ groups. There was also no significant difference in operative time, $\mathrm{t}(33)$ $=1.31, p=.19$ for surgeon experience of 10 or more years, although the ipsilateral group had a shorter operative duration by 34 minutes.
A total of 38 patients were included in the computations for hearing gain. Of these, 22 patients were in the contralateral $(C)$ group and 16 were in the ipsilateral (I) group. There was no statistically significant difference, $\mathrm{t}(36)=-0.72, \mathrm{p}=.47$, in overall mean hearing gain among patients in the contralateral $(M=+2.22 \mathrm{~dB}, \mathrm{SD}=10.54)$ and ipsilateral $(M=+5.12 \mathrm{~dB}, \mathrm{SD}=14.26)$ groups. Those procedures operated on by surgeons with less than 10 years of experience, the contralateral $(M=$ $+4.06 \mathrm{~dB}, \mathrm{SD}=13.41)$ and ipsilateral $(M=+2.29 \mathrm{~dB}, \mathrm{SD}=16.88)$ hearing gains did not differ significantly, $t(18)=0.26, p=.79$. Although the difference was also not statistically significant, $t(16)=-1.94, p=.07$ for contralateral $(M=0.00, S D=5.43)$ and ipsilateral $(M=+7.95 \mathrm{~dB}, \mathrm{SD}=$ 11.52) procedures performed by surgeons with experience of 10 years or more a mean hearing gain of $+7 \mathrm{~dB}$ in the ipsilateral group compared to $0 \mathrm{~dB}$ in the contralateral group was notable, as it was closer to the $10 \mathrm{~dB}$ pre- and post-operative air-bone gap difference cut-off for significant hearing improvement.

\section{DISCUSSION}

This study found no significant difference in operative duration or hearing improvement among patients whose surgical site was ipsilateral the surgeon's dominant hand compared to those whose surgical site was contralateral the surgeon's dominant hand, even when surgeon experience was taken into consideration.

However, the shorter overall operative duration of 20 minutes in the ipsilateral group, increasing to 37 minutes among surgeons with 10 or more years of experience cannot be overlooked. Although there was also no significant difference between surgeon handedness and surgical site laterality in terms of hearing gain, the ipsilateral group operated on by surgeons with 10 or more years of experience attained hearing gains better by $7 \mathrm{~dB}$. Because of these, my hypothesis that operative duration should be shorter and hearing gain will be better when operative ears are ipsilateral to the surgeon's dominant hand, and that these outcomes may be further improved with surgeon experience greater than ten years, cannot just be dismissed outright.

Although they are not statistically significant, these findings are clinically important to the surgeon, because they can help gauge success rates and estimate duration of operations. They may also be relevant to the patient because shorter operative times would mean less exposure to anesthetics and other intraoperative medications. Hearing gain is considered significant if the pre-operative and post-operative air-bone gap difference is $10 \mathrm{~dB}$ or higher.7 Since the ipsilateral group came closer to this value, there may yet be a potential advantage associated with operating on the ear ipsilateral to the surgeon's dominant hand.

The findings of this study echo those of previous studies in other surgical fields operating on paired body parts. A right-handed surgeon 
operating on the left side of the patient had higher odds of having a poor outcome than when operating on the right, ${ }^{3}$ favoring procedures done on the side ipsilateral to the surgeon's dominant hand. There was also evidence that operating ipsilateral to the surgeon's dominant hand resulted in a better surgical outcome. ${ }^{4}$ The additional impact of surgeon experience seems to attenuate the effects of surgeon handedness and laterality of the surgical site alone. ${ }^{5}$ Although statistically insignificant, this may be suggested by our findings of shorter durations and higher hearing gains among contralateral and ipsilateral groups for procedures performed by more experienced surgeons. A more extensive series or perhaps a future randomized controlled trial may shed more light on this issue.

Perhaps the reason for the advantage of operating on the ear ipsilateral the surgeon's dominant hand may relate to the ergonomically easier access and maneuvering of instruments in the ipsilateral middle ear and mastoid. In contrast, operating on the side contralateral to the surgeon's dominant hand may require hyper-adducting their wrist to manipulate and dissect the middle ear, especially in the attic and the sinodural angle. A future simulation study may demonstrate whether this is so.

This was a retrospective cohort study wherein records of patients were reviewed. Causation of phenomena such as change in operative duration and improvement in post-operative hearing, cannot be determined although association may be. An important limitation of the study is that our obtained sample size, while adequate per computed sample size, was not consistent for both outcomes. Half of the patients lacked records of post-operative audiograms for various reasons (the surgeons did not request them, the patients did not have the audiogram done, or the patients did not return for follow-up after the procedure). This affects our results and conclusions for this outcome. The otologic procedures varied, and perhaps to have a more consistent determination of association, one type of surgical procedure should be included (e.g., tympanoplasty only or type 3 mastoidectomy with tympanoplasty only). Also, a surgeon's experience on otologic surgery varies widely since few perform the same type of surgery with the same frequency despite the number of years of being a boardcertified otolaryngologist. Future studies could stratify surgeons by the number of tympanomastoidectomies done per year as to better gauge their skill or experience. Another limitation is that complications were not recorded in the study, and this is an important variable to consider when measuring the surgical outcome of operative duration in future research.

To the best of my knowledge, this is the first study to examine how surgeon handedness, surgeon experience, and surgical site laterality may impact ear surgery outcomes. Although a measure of efficiency may have been approximated by the number of minutes per procedure, efficacy cannot be adequately measured because of the numerous study limitations.

In conclusion, this study did not prove that regardless of surgeon experience, right-handed surgeons operating on the right ear and left-handed surgeons operating on the left ear have better ear surgery outcomes of operative duration and hearing improvement compared to right-handed surgeons operating on the left ear and lefthanded surgeons operating on the right ear of the patient. However, future studies on larger samples with more complete data may yet demonstrate this effect.

\section{ACKNOWLEDGEMENTS}

I would like to thank my adviser Dr. Jose Acuin, for overall supervision of my research and Dr. Patrick Pardo for providing feedback on my manuscript; our department chairman Dr. Ramon Ramos III, for general support in this endeavor; and Ms. Loida Aquino and Mr. Brian Jay Feranil for assistance in data collection.

\section{REFERENCES}

1. McManus IC. The history and geography of human handedness. In: Sommer, Iris EC and Rene S Kahn. Language Lateralization and Psychosis. Cambridge: Cambridge University Press, 2009. P. 37-57. DOI: https://doi.org/10.1017/CBO9780511576744.004.

2. Zaghloul M, Saguib J, Al-Mazrou A, Saguib N. A Qualitative Assessment of the impact of handedness among left-handed surgeons in Saudi Arabia. Laterality. 2018 Jan;23(1):39-50. DOI: 10.1080/1357650X.2017.1309049; PubMed PMID: 28363266.

3. Mehta S, Lotke PA. Impact of Surgeon Handedness and Laterality on Outcomes of Total Knee Arthroplasties: Should Right-Handed Surgeons Do Only Right TKAs? Am Journal Orthop. 2007 Oct;36(10):530-3. PubMed PMID: 18033564.

4. Pennington N, Redmond A, Stewart T, Stone M. The impact of surgeon handedness in total hip replacement. Ann R Coll Surg Engl. 2014 Sep; 96(6): 437-441. DOI: 10.1308/003588414X1394618 4902488; PubMed PMID: 25198975; PubMed Central PMCID: PMC4474195.

5. Luvisa K, Fan KL, Black CK, Wirth P, Won Lee D, Del Corral G, et al. Does surgeon handedness or experience predict immediate complications after mastectomy? A critical examination of outcomes in a single health system. The Breast Journal. 2019 Aug; 26(10) 1-8. DOI: 10.1111/ tbj.13487.

6. Naunheim MR, Le A, Dedmon MM, Franco RA, Anderson J, Song PC. The effect of handedness and laterality in a microlaryngeal surgery simulator. Am J Otolaryngol. 2017 Jul-Aug; 38(4):472 474. DOI: 10.1016/j.amjoto.2017.04.009; PubMed PMID: 28449823.

7. Gupta S, Parmod K, Sehgal S, Gupta N. Review of Parameters Used To Assess Hearing Improvement in Tympanoplasty. IOSR-JDMS. 2016 Feb; 15 (2 ver X): 122-128. DOI: 10.9790/085315210122128. 